\title{
克酮酸菁染料研究进展
}

\author{
张守金鈢杨小兵* 温晓红 丁松涛王安邦 \\ (防化研究院国民核生化灾害防护国家重点实验室 北京 100191)
}

\begin{abstract}
摘要 克酮酸菁染料是有机功能染料菁类染料中的重要成员, 由于其在近红外区具有吸收峰强烈且尖锐、摩尔吸光系 数大、光热稳定性好等特点, 近年来引起了人们的关注. 概括和总结了克酮酸菁染料的结构特点、物化性质及其合成 途径，同时介绍了它在光学材料、生物和医学等领域潜在的应用价值.
\end{abstract}

关键词 克酮酸菁染料; 结构; 性质; 合成; 应用

\section{Progress in the Studies of Croconium Dyes}

Zhang, Shouxin $\quad$ Yang, Xiaobing* Wen, Xiaohong $\quad$ Ding, Songtao Wang, Anbang

(State Key Laboratory of Nuclear Biological and Chemical Protection for Civilian, Research Institute of Chemical Defense, Beijing 100191)

\begin{abstract}
Croconium dye is an important part in the cyanine family used as organic function dye, which has been attracted extensive attention recently because of its strong and sharp absorption, large molar absorption coefficient and good photochemical stability in the near-infrared region. The unique structure, and physical and chemical properties, together with the recent progress in the preparation methods of croconium dyes are reviewed. The promising application in the fields of optical materials, biology, medicine, etc. is also introduced.
\end{abstract}

Keywords croconium dye; structure; property; synthesis; application

近年来, 有机功能染料引起了人们的极大关注. 有 机功能染料在光学领域应用广泛, 如光数据存储、光学 转换和太阳能电池等方面, 同时在苂光检测、抗癌剂、 生物分子标记等生物和医学方面也有着广泛的应用. 克 酮酸菁染料是目前正在研究的一种有机功能染料, 它在 近红外区吸收峰强烈且尖锐、摩尔吸光系数高、苂光发 射能力强、光热稳定性好, 这些特点使其在激光存储、 太阳能电池、非线性光学和光动力学疗法等方面具有广 阔的应用前景 ${ }^{[1 \sim 7]}$. 目前, 国内外的研究对象主要还是 对称型克酮酸菁染料, 相对而言不对称型的克酮酸菁染 料报道很少. 本文总结了克酩酸菁染料的结构、物化性 质、合成途径及其应用方面的研究进展.

\section{1 克酮酸菁染料的结构与性质}

\section{1 结构及分类}

克酮酸菁染料属于菁染料的一种, 是由克酮酸与具 有供电子能力的基团缩合而成, 可以看作是菁染料中心
的次甲基被相应的克酩酸取代而成 ${ }^{[8]}$. 克酮酸菁染料分 子一般由推电子-拉电子-推电子(D-A-D)基团构成, 中 心的克酮酸是强吸电子基团，它与两端连接的供电子基 团组成大型的 $\pi$ 共轭体系 ${ }^{[9]}$. 染料分子中含有介离子结 构 1 (2-氧代丙烷-1,3-二基或三亚甲基-2-酚盐, Chart 1) 作为电子受体，是一种介离子化合物. 介离子化合物是 偶级五元或六元环状化合物，化合物中的正负电荷是离 域的, 大多数情况下正电荷和环上的原子相连, 而负电 荷和环上的原子或环外的原子相连. 分子中的介离子结 构使得克酮酸菁染料产生强烈的 $\pi-\pi$ 跃迁，使电荷在整 个分子上离域 ${ }^{[10]}$.

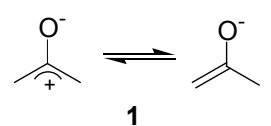

Chart 1

介离子结构 1 两侧可引入不同取代基团, 这些取代

\footnotetext{
*E-mail: xbyoung2001@163.com,xbyang@pku.edu.cn

Received August 4, 2013; revised September 3, 2013; published online September 17, 2013.

Project supported by the National Natural Science Foundation of China (No. 21002123).

国家自然科学基金(No. 21002123)资助项目.
} 
基的引入会引起染料分子 $\pi-\pi$ 能级跃迁的改变, 导致染 料分子的最大吸收波长产生一定范围的波动. 根据与克 酮酸两端连接的取代基的不同，可以分为对称型和不对 称型(结构 2, Eq. 1). 若 $R^{1}$ 和 $R^{2}$ 相同, 则为对称型克酮 酸菁染料; 若 $R^{1}$ 和 $R^{2}$ 不相同，则为不对称型克酮酸菁 染料. 通常情况下, 取代基的结构为具有供电子能力的 芳环及其衍生物, 如苯胺、吡咯、噻吩、喹啉和宷类等.<smiles>O=c1c(O)c(O)c(=O)c1=O</smiles><smiles>[R]C1=C([O-])C([R])C(=O)C1=O</smiles>

\section{2 物理化学性质}

\subsection{1 近红外吸收性能}

克酮酸菁染料的吸收光谱具有溶致变色迁移吸收 效应 ${ }^{[11]}$, 即它的最大吸收波长随着溶剂极性变化而发 生 “红移” 或 “蓝移” 。红移是指染料的最大吸收波长 向更高波段移动; 而蓝移正好相反, 最大吸收波长向更 低波段移动. 通常克酮酸菁染料在近红外区 $(750 \sim 1400$ $\mathrm{nm})$ 具有强烈且尖锐的吸收峰和较高的摩尔吸光系数, 与相应的方酸菁染料相比, 克酩酸环比方酸环具有更强 的吸电子能力, 所以克酮酸菁染料的最大吸收波长比相 同结构的方酸菁染料要红移 $100 \mathrm{~nm}$ 左右 ${ }^{[12,13]}$.

化合物 3 (Chart 2) 是一种新合成的克酩酸菁染料, 在克酩酸两侧引入了取代氨基苯二酚基团, 该染料的最 大吸收波长在 $860 \mathrm{~nm}$ 左右, 但是它却容易异构化成化 合物 4 [di(benzofuranonyl)methanolate, 简称 BM 衍生物] (Chart 2), 是由两个苯并呋喃基团连接一个甲氧基构成, 它的最大吸收波长位于 $1100 \mathrm{~nm}$ 左右, 与 $\mathbf{3}$ 相比最大吸 收波长红移超过 $200 \mathrm{~nm}^{[14 \sim 17]}$.<smiles>[R]C=[N+]=C1C=C(O)C(=C2C(=O)C(=O)C(c3c(O)cc(N([R10])C)cc3O)=C2[O-])C(O)=C1</smiles>

3

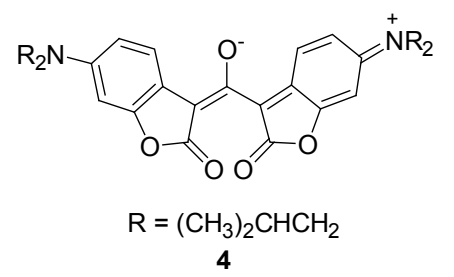

Chart 2

Langhals ${ }^{[18]}$ 分析了 $\mathrm{BM}$ 衍生物产生强烈红移的原因, 该课题组基于 Konig 和 Ismaisky 的 D- $\pi-A-\pi$-D (D 是给 电子基团, $\mathrm{A}$ 是吸电子基团)经验模型, 推测碳正离子是 电子受体, BM 衍生物在更长波长区有吸收是由于共轭
体系的增大. 当电子受体即吸电子基团被方酸(形式上 带有两个正电荷)取代时，吸收波长就出现红移; 而当 方酸被具有三个正电荷的克酮酸取代时，会有更大的红 移. BM 衍生物出现红移是由于内酯环上的两个羰基作 为超级吸电子基团, 胺基作为供电子基团. 根据这个模 型, 可以推测, 如果分子结构中的供电子基团被超级供 电子基团取代, 将会产生更大的红移. Meier 等 ${ }^{[19,20]}$ 认为 BM 染料中心的三亚甲基-2-酚盐结构是电子受体，发生 红移主要是由于供电子基团的变化, 使得 $\pi$ 共轭体系得 到拓展. Tatsuura 等 ${ }^{[21]}$ 推测其红移的两个主要原因, 一 个是分子中心区域 $\pi$ 电子的集中和内酯基团作为超级吸 电子基团, 另一个是苯并呋喃基团整体立体构型的扭 转, 其扭转角度的大小与吸收波长的峰值紧密相关.

\subsection{2 自缔合性能}

克酮酸菁染料一般易溶于有机溶剂，如四氢呋喃、 二氯甲烷、三氯甲烷、乙腈、甲醇、丙酮、乙醇、DMF、 乙酸等, 较难溶于水. 克酩酸菁染料属于菁染料中的一 大类. 一般而言, 菁染料分子间有很强的范德华力, 在 溶液中往往会产生自缔合现象, 形成聚集体. 在不同的 溶液及固体介质中, 克酮酸菁染料也会形成聚集体, 聚 集体的吸收峰与单体的有很大不同. 研究表明 ${ }^{[22 ~ 24]}$, 聚 集体根据偶极跃迁的方向分可为两种类型，一种类型是 $\mathrm{H}-$ 聚集体，分子以 “头接头” 的偶极跃迁方式排列，与 单体相比表现出 “蓝移” 的吸收特性, 即吸收波长较原 吸收波长小; 另一种类型称之为 $\mathrm{J}$-聚集体，分子以 “头 接尾” 的偶极跃迁方式排列, 通常它会有一个尖锐而强 烈的红移吸收带, 并会产生共振荧光, 而 $\mathrm{H}$-聚集体是非 荧光的. 通常情况下, 利用这类化合物的自缔合性能可 以区分染料分子在溶液中的聚集状态.

\section{2 .3 光稳定性}

光照条件下, 染料分子的结构会遭到破坏，从而引 起变色或褪色. 菁染料的褪色主要是染料分子与光发生 氧化反应引起的, 具体机理有电子转移和能量转移两种 机制(Scheme 1). 在能量转移过程中, 染料分子受到光 照射后激发成三线态, 再与氧反应生成单线态氧, 单线 态氧与染料分子进行氧化反应使染料褪色. 在电子转移 机制中，染料分子受到光照射激发成单线态，再与氧反 应生成超氧负离子, 超氧负离子与染料分子进行氧化反 应使染料裖色. 因此, 如果染料分子的结构能延缓这个 过程的变化, 就会增加其光稳定性. 与中心是多甲川链 的直链菁染料相比，克酮酸菁染料的中心是环状体系的 共轭单元，提高了分子的刚性和空间位阻，不利于单线 态氧的进攻, 因此, 克酮酸菁染料比一般的菁染料更稳 定 ${ }^{[25]}$. 


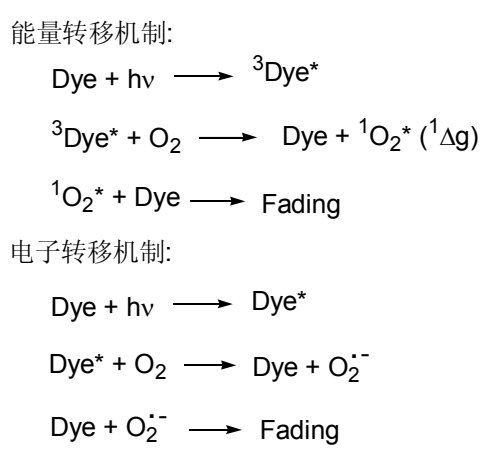

Scheme 1

\section{2 克酮酸菁染料的合成途径}

1966 年, Sprenger 等 ${ }^{[26]}$ 提出, 以方酸和 2 equiv. 的亲 核试剂在回流条件下反应，所用溶剂通常为甲苯/正丁 醇, 用吡啶或喹啉等作催化剂, 通过 Dean-Stark 分水器 除去缩合反应中生成的水, 产物就是方酸菁染料. 克酥 酸菁染料和方酸菁染料的合成方法基本一样 ${ }^{[27]}$ ，也是 由克酮酸与相应的亲核试剂缩合而成(Scheme 2).
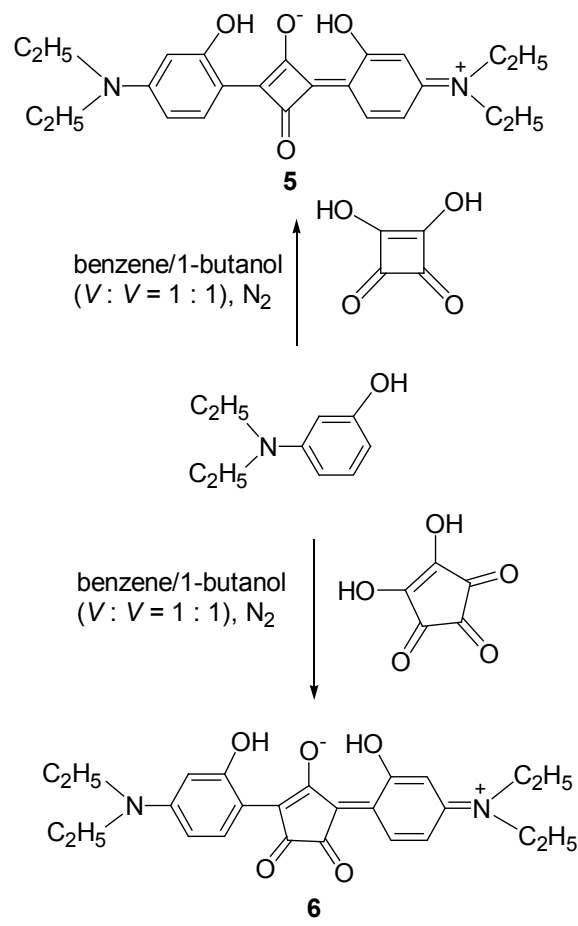

Scheme 2

克酮酸菁染料经典的合成方法是通过克酮酸与 2 equiv. 的亲核试剂, 在回流条件下反应, 溶剂通常为体 积比为 $1: 1$ 的甲苯/正丁醇或苯/正丁醇等混合溶剂, 也 有报道采用乙醇或乙酸等其它溶剂作为反应介质; 亲核 试剂一般为喹哪啶、吲哚、噻吩、苯并噻吩、苯胺等供 电子基团(Chart 3). 反应结束后, 将反应液冷却至室温, 过滤得到沉淀, 然后用乙醚、甲醇或正己烷等洗涤沉淀, 或通过柱色谱分离, 得到产物 ${ }^{[28 ~ 32]}$.<smiles>[X]c1ccc(C)[n+]([Y])c1</smiles><smiles>[X]c1ccsc1I</smiles><smiles>[X]C1C2C=CC(C)C1N([Y])C(C)=C2</smiles><smiles>[R16]Nc1ccc(C)c(O)c1</smiles><smiles>CC1c2ccccc2-c2ccccc21</smiles>

Chart 3

1993 年, Keil 等 ${ }^{[29]}$ 用噻吩取代基和克酮酸在体积比 1：1的正丁醇/甲苯混合溶剂中回流反应，得到化合物 7 和 8 (Scheme 3), 产率分别为 55\% 70\%和 45\% 55\%. 化合物 7 和 8 是深色、高熔点 $\left(>360{ }^{\circ} \mathrm{C}\right)$ 的微晶固体，近 红外区吸收峰很强, 但是在许多有机溶剂中溶解度很 低. 表 1 和表 2 给出了化合物 7 和 8 在二氯甲烷中的最 大吸收波长.
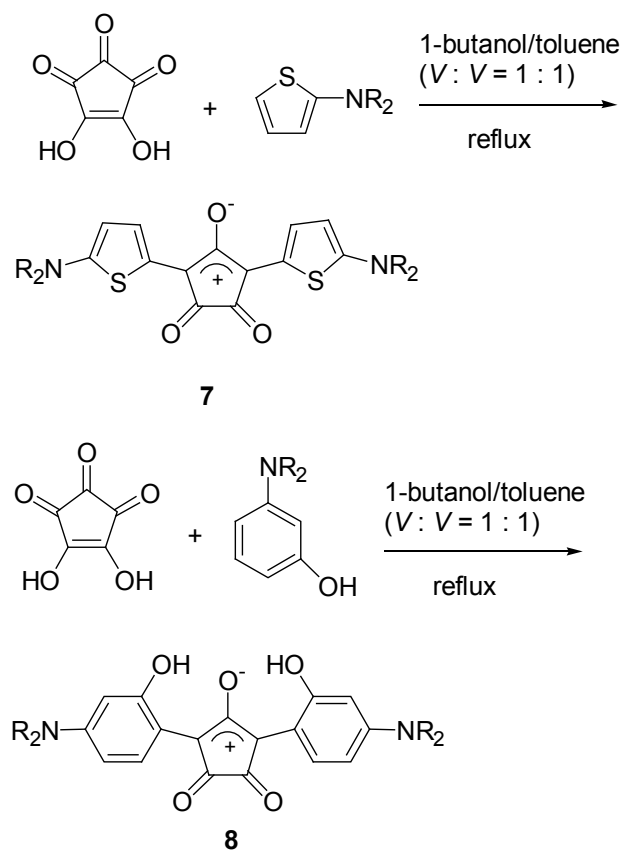

Scheme 3

表 1 化合物 7 在二氯甲烷中的最大吸收波长

Table 1 Maximum absorption wavelength of compound 7 in DCM

\begin{tabular}{ccc}
\hline Compound & $\mathrm{R}$ & $\lambda_{\max } / \mathrm{nm}$ \\
\hline $\mathbf{7 a}$ & $\mathrm{Me}$ & 786 \\
$\mathbf{7 b}$ & 783 \\
$\mathbf{7 c}$ & & 798 \\
$7 \mathbf{7 d}$ & & 792.5 \\
\hline
\end{tabular}


表 2 化合物 8 在二氯甲烷中的最大吸收波长

Table 2 Maximum absorption wavelength of compound $\mathbf{8}$ in DCM

\begin{tabular}{ccc}
\hline Compound & $\mathrm{R}$ & $\lambda_{\max } / \mathrm{nm}$ \\
\hline $\mathbf{8 a}$ & $\mathrm{Me}$ & 817 \\
$\mathbf{8 b}$ & $\mathrm{Et}$ & 822 \\
\hline
\end{tabular}

2000 年, Simard 等 ${ }^{[28]}$ 用氧族吡喃鎓盐与克酮酸在甲 醇和吡啶加热条件下反应, 经过冷却、过滤和柱色谱纯 化, 得到化合物 9 (Eq. 2). 该研究小组发现制备 $9 b$ 的最 佳温度是 $80{ }^{\circ} \mathrm{C}$, 而 $9 \mathbf{a}, 9 \mathbf{c}$ 和 $9 \mathbf{d}$ 是 $100{ }^{\circ} \mathrm{C}$, 在 $100{ }^{\circ} \mathrm{C}$ 下 9b 的产率却只有 $12 \%$. 化合物 $9 \mathrm{a}$ 在有机薄膜中具有很 好的溶解能力, 已经在热写显示、电子照排和光学记录 等领域得到应用，而有关 $9 b, 9 c$ 和 $9 d$ 的应用没有查阅到 相关报道. 化合物 9 的最大吸收波长覆盖大部分激光发 射的范围, 从 $820 \mathrm{~nm}$ 的镓砷二极管激光到 $1064 \mathrm{~nm}$ 的钕 激光, 因此这类化合物有望作为染料激光器中的工作介 质. 表 3 是化合物 9 的最大吸收波长和产率.

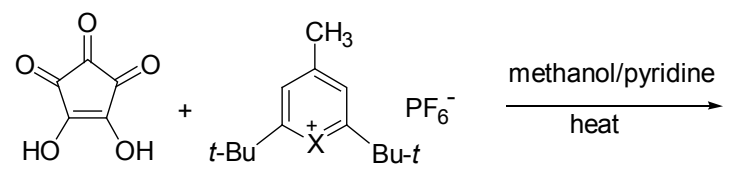<smiles>[X]c1cc(C=c2c(=O)c(=O)c(=Cc3c[Z](Br)cc(Br)c3)c2=O)cc(C(C)(C)C)c1</smiles>

表 3 化合物 9 在二氯甲烷中的最大吸收波长

Table 3 Maximum absorption wavelength and yields of compound 9 in DCM

\begin{tabular}{cccc}
\hline Compound & $\mathrm{X}$ & $\lambda_{\max } / \mathrm{nm}$ & Yield/\% \\
\hline 9a & $\mathrm{O}$ & 845 & 75 \\
9b & $\mathrm{S}$ & 950 & 63 \\
9c & $\mathrm{Se}$ & 999 & 81 \\
9d & $\mathrm{Te}$ & 1081 & 56 \\
\hline
\end{tabular}

2002 年, Eninas 等 ${ }^{[32]}$ 用苯并噻吩基团与克酮酸在甲 苯和正丁醇混合溶剂中回流反应, 将最终反应后的混合 物在一 $20{ }^{\circ} \mathrm{C}$ 下冷却过夜, 过滤后得到的晶体产物用乙 醚冲洗, 通过甲醇重结晶, 制备出化合物 10 (Eq. 3), 产 率为 76\%. 化合物 $\mathbf{1 0}$ 的最大吸收波长位于 $780 \mathrm{~nm}$ 左右 的近红外区, 且随着 $\mathrm{pH}$ 的增大而蓝移, 其主要原因是 $\mathrm{N}$ 原子发生去质子化, 阻碍了电荷离域. 利用化合物 10 的上述特性, 同时结合该类染料在聚合物薄膜中具备良 好的溶解性等特性, 可望将其作为光学 $\mathrm{pH}$ 敏感膜近红 外元器件中关键的功能材料. 表 4 列出了化合物 $\mathbf{1 0}$ 的产 率及其在不同酸、碱性条件下的最大吸收波长.
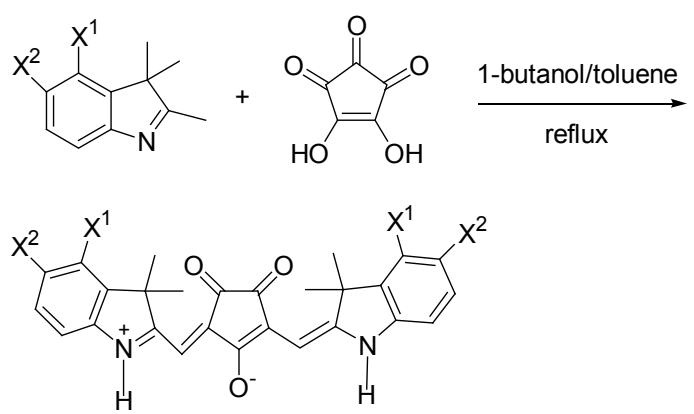

10

表 4 化合物 $\mathbf{1 0}$ 的产率和在乙醇中不同酸碱条件下的最大吸 收波长

Table 4 Yields of compound $\mathbf{1 0}$ and maximum absorption wavelength in acid and basic ethanol solution

\begin{tabular}{|c|c|c|c|c|c|}
\hline \multirow{2}{*}{ Compound } & \multirow{2}{*}{$X^{1}$} & \multirow{2}{*}{$X^{2}$} & \multicolumn{2}{|c|}{$\lambda_{\max } / \mathrm{nm}$} & \multirow{2}{*}{ Yield/\% } \\
\hline & & & Acid & Basic & \\
\hline $10 \mathrm{a}$ & $\mathrm{H}$ & $\mathrm{H}$ & 759 & 626 & 76 \\
\hline $10 \mathrm{~b}$ & $\mathrm{H}$ & $\mathrm{C}_{4} \mathrm{H}_{9}$ & 775 & 624.5 & 63 \\
\hline $10 \mathrm{c}$ & $\mathrm{H}$ & $\mathrm{OCH}_{3}$ & 793.5 & 638.5 & 36 \\
\hline 10d & $\mathrm{H}$ & $\mathrm{NO}_{2}$ & 793 & 630 & 70 \\
\hline $10 \mathrm{e}$ & $\mathrm{H}$ & $\mathrm{NHCOCH}_{3}$ & 798.5 & 639.5 & 35 \\
\hline
\end{tabular}

2003 年，Tian 等 ${ }^{[14]}$ 用胺基苯二酚与克酮酸在甲苯/ 正丁醇体积比为 $3: 1$ 的回流条件下反应, 意外得到了 化合物 4 (Scheme 4), 产率为 7.4\%. 它的最大吸收波长 在 $1100 \mathrm{~nm}$ 左右, 而且具有很高的摩尔吸光系数. 它是 由克酮酸菁染料 $\mathbf{3}$ 异构化而成，化合物 $\mathbf{3}$ 的最大吸收波 长是 $860 \mathrm{~nm}$ 左右, Langhals 指出化合物 4 产生巨大红移 的原因是中心的介离子结构产生了一个超级吸电子基 团 ${ }^{[18]}$.
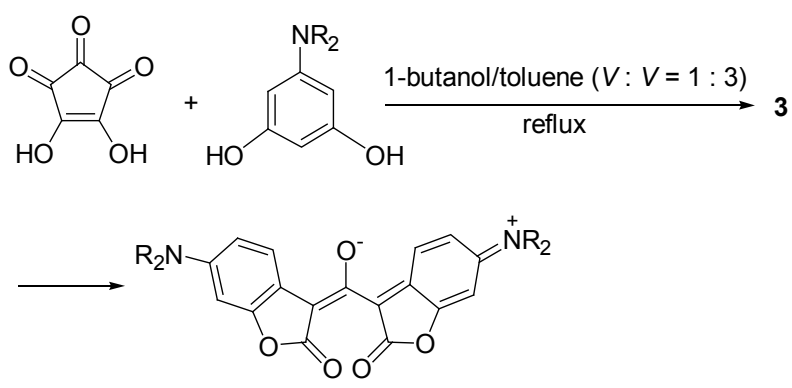

$$
\stackrel{4}{\mathbf{R}}=\left(\mathrm{CH}_{3}\right)_{2} \mathrm{CHCH}_{2}
$$

\section{Scheme 4}

2004 年, 彭孝军和王丽秋 ${ }^{[33]}$ 报道, 由 2,3,3-三甲基$3 H$-吲哚磺酸衍生物与克酮酸在苯和正丁醇混合溶剂中 回流反应，冷却反应液后，用乙醚稀释，过滤得到绿色 固体，再经过柱色谱纯化，最终得到水溶性 $3 H$-吲哚克 酮酸菁染料 11 (Chart 4). 该研究小组称由于吲哚环 5 位 上磺酸基的存在，增强了分子的水溶性；同时，通过 $\mathrm{N}$ 原子上取代基的更换，可使染料的苂光性能有所改善， 
利用该性能可以用作生物荧光标示剂.<smiles>[R7]c1c([R])c([R])c2c(c1[R])c([Z])c(/C=c1\c(=O)c(=O)/c(=C\C3=[N+](Cc4ccccc4)c4ccc(S(=O)(=O)[O-])cc4C3(C)C)c1=O)n2[R]</smiles>

$\mathrm{Z}=\mathrm{O}, \mathrm{S}, \mathrm{Se}, \mathrm{Te}, \mathrm{CR}^{2} \mathrm{R}^{3}\left(\mathrm{R}^{2}, \mathrm{R}^{3}=\mathrm{H}, \mathrm{CH}_{3}, \mathrm{CF}_{3}, \mathrm{C}_{2} \mathrm{H}_{5}\right)$

$\mathrm{R}^{1}=\left(\mathrm{CH}_{2}\right)_{m} \mathrm{CH}_{3},\left(\mathrm{CH}_{2}\right)_{m} \mathrm{COOH},\left(\mathrm{CH}_{2}\right)_{m} \mathrm{SO}_{3}, m=0 \sim 18$;<smiles>CCc1ccc(C(=O)O)cc1</smiles>

$\mathrm{R}^{4}, \mathrm{R}^{5}, \mathrm{R}^{6}, \mathrm{R}^{7}=\mathrm{H}, \mathrm{F},\left(\mathrm{CH}_{2}\right)_{p} \mathrm{CF}_{3},\left(\mathrm{CH}_{2}\right)_{p} \mathrm{CN}, \mathrm{SO}_{3}, \mathrm{SO}_{3} \mathrm{H},\left(\mathrm{CH}_{2}\right)_{p} \mathrm{CH}_{3}$, $\mathrm{OH}, \mathrm{O}\left(\mathrm{CH}_{2}\right)_{p} \mathrm{CH}_{3},\left(\mathrm{CH}_{2}\right)_{p} \mathrm{COOH}, p=0 \sim 4$

\section{Chart 4}

2007 年, Avirah 等 ${ }^{[31]}$ 先用喹哪啶与碘甲烷成盐, 再 与克酮酸进行缩合, 以乙醇作溶剂, 喹啉作催化剂, 加 热至 $80{ }^{\circ} \mathrm{C}$ 反应, 得到了喹哪啶型克酮酸菁染料 $\mathbf{1 2}$ (Scheme 5), 产率为 $80 \% \sim 90 \%$. 它们的最大吸收波长 在 800 900 nm之间, 12a, 12b 和 12c 在普通有机溶剂中 溶解度较小, 而 $12 \mathrm{~d}$ 在三氯甲烷和四氢呋喃中的溶解度 有所提高. 研究小组发现, 这些染料在近红外区有尖锐 而强烈的吸收峰, 并且能与金属离子, 如 $\mathrm{Zn}^{2+}, \mathrm{Pb}^{2+}$, $\mathrm{Cd}^{2+}$ 等络合(Eq. 4), 随着金属离子浓度增大, 最大吸收 波长会蓝移, 苂光光谱也会随之变化, 因此, 这类克酮 酸菁染料可望被设计成红外金属离子传感器, 以及用作 光动力学疗法中的光敏剂.

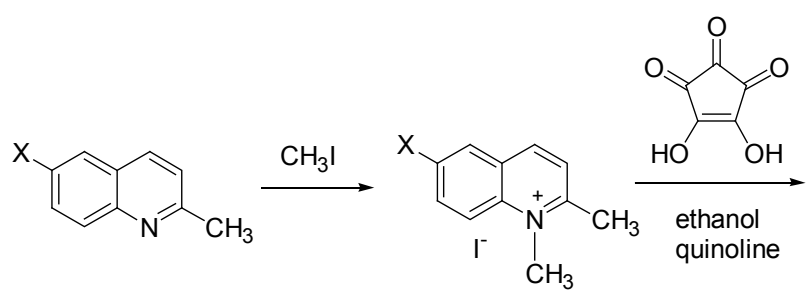<smiles>[X]C1=CC=C2C(=CC=C(C=C3C(=O)C(=O)C(=Cc4ccc5cc([X])ccc5[n+]4C)C3[O-])N2C)C=C1[X]</smiles>

12

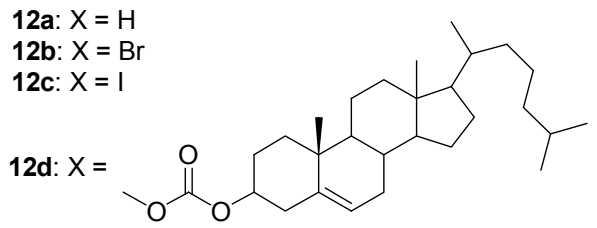

Scheme 5<smiles></smiles><smiles>[M]OC1=C(/C=C2\C=Cc3cc([X])ccc3N2C)C(=O)/C(=C/c2ccc3cc([X])ccc3[n+]2C)O1</smiles><smiles></smiles>

2008 年, Song 等 ${ }^{[34]}$ 用哌啶衍生物与克酮酸反应，在 甲苯与正丁醇溶剂体系中回流缩合，反应得到一种新型 水溶性克酮酸菁染料 $\mathbf{1 3}$ (Eq. 5), 产率为 $95 \%$. 化合物 $\mathbf{1 3}$ 在 $783 \mathrm{~nm}$ 处呈现一个尖锐而强烈的吸收峰, 且在可见 光区几乎没有吸收峰，在水、甲醇和塑料薄膜中具有很 好的光稳定性. 由于它具有良好的水溶性，通过水性旋 涂过程，可以制造近红外激光系统里的滤光片，而且避 免了传统的有机溶剂旋涂法带来的环境危害.

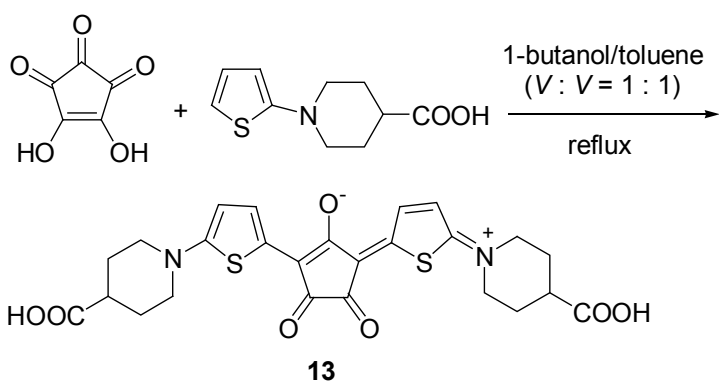

2012 年, Kurdiukova 等 ${ }^{[30]}$ 采用二苯并五环衍生物与 克酩酸在乙酸酤加热反应，得到克酮酸菁染料 14 (Eq. 6), 产率 64\%. 克酮酸菁染料 14 在 DMF 中的最大吸收<smiles>CC1C2Cc3cc([N+](=O)[O-])cc([N+](=O)[O-])c3-c3c(cc([N+](=O)[O-])cc3[N+](=O)[O-])C12</smiles>

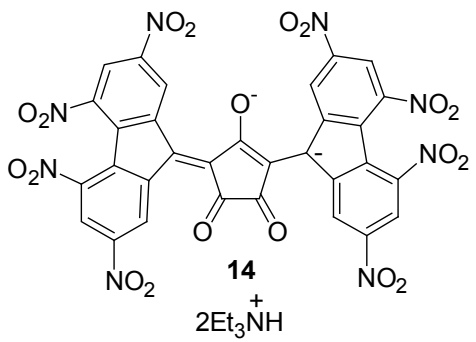


波长为 $1052 \mathrm{~nm}$, 与其它传统只在近红外区具有强烈吸 收的克酮酸菁染料相比, 它在可见光区和近红外区都有 非常强烈的吸收.

\section{3 克酮酸菁染料的应用}

\section{1 光动力学疗法}

近年来, 光动力学疗法在医学领域受到了广泛关 注, 尤其在肿瘤治疗方面发展迅速. 它的治疗原理是根 据癌细胞的特异性, 向其注入特定的对光敏感的物质即 光敏剂, 它本身无毒, 且能在人体内实现较长时间的停 留; 当以一种特定波长的光照射时, 光敏剂分子发生能 级跃迁, 通过能量转移过程与氧相互作用, 产生具有毒 性作用的单线态氧, 从而破坏杀死癌细胞 ${ }^{[35]}$. 克酮酸菁 染料的最大吸收波长可以扩展到近红外区，涵盖的波长 范围宽, 对光的吸收强度高, 以该类染料作为光敏剂, 可以增加穿透组织的深度, 使作用的范围大大增加.

\section{2 非线性光学材料}

随着科技的发展, 非线性光学材料引起了人们的关 注, 这类材料在光学通讯、数据存储、全息摄影和谐波 发生器等方面有广阔的应用前景 ${ }^{[36]}$. 光学装置中所用 的非线性光学材料, 要求具有较高的非线性光学密度、 较低的光学损失和快速响应时间等特性 ${ }^{[37]}$. 克酮酸菁 染料在可见一近红外区有强烈的吸收和较高的摩尔吸光 系数，通过分子结构的修饰可以调节其最大吸收波长和 溶解性，使其在非线性光学领域有着巨大的应用潜力.

化合物 10a 是一种吲哚类的克酮酸菁染料, 溶解性 能优良, 李忠玉等 ${ }^{[37]}$ 采用旋涂制模法研究了它的三阶 非线性光学性质. 他们指出 $10 \mathrm{a}$ 在有机溶剂中的吸收光 谱呈现负溶致变色效应，即最大吸收波长随溶剂极性增 大而移向短波; 而在薄膜中, 克酩酸染料的峰值吸收与 在溶液中相比发生红移, 吸收峰也变宽, 这是由于克酮 酸染料分子在膜中形成了聚集体. 他们采用简并四波混 频法测试了 $10 \mathrm{a}$ 的三阶非线性光学性质, 得出 $10 \mathrm{a}$ 具有 大的三阶非线性极化率和快速的非线性光学响应时间, 性能优于大部分材料. 因此, 克酮酸菁染料 10a 在非线 性光学材料领域有潜在的应用价值.

\section{3 分析检测}

克酮酸菁染料在近红外区通常具有很强的荧光发 射, 所以它可以应用于化学传感器中, 成为在生物和医 学等领域作为一种检测手段. 化合物 $\mathbf{1 0}$ 是一种新型的 吲哚类克酮酸菁染料, 其最大吸收波长能随着溶液 $\mathrm{pH}$ 的变化而变化, 因此可以用作光传感器中的 $\mathrm{pH}$ 敏感元 件 ${ }^{[33]}$. 化合物 12d 具有是一种喹哪啶型克酩酸菁染料, 中心克酮酸环上的羰基氧能与二价金属离子(如 $\mathrm{Zn}^{2+}$,
$\left.\mathrm{Mg}^{2+}\right)$ 产生很强的鳌合作用，使其荧光发射产生显著的 变化，因此，它可以用来检测某些二价金属离子 ${ }^{[32]}$. 化 合物 15 (Chart 5) 是一种苯胺类的克酮酸菁染料，它可以 作为近红外比色化学剂量计来检测半胱氨酸以及氭根 离子等，与相应的方酸菁染料相比，它具有更高的灵敏 度和更快的响应时间 ${ }^{[28]}$.

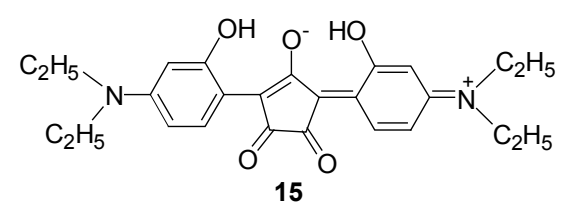

Chart 5

\section{4 染料敏化太阳能电池}

染料敏化太阳能电池(DSSC)的工作原理是利用光 敏材料吸收光能后发生光电转换，它具有成本低、制备 简单、稳定性好等优点 ${ }^{[38]}$, 已成为研究的热点. 目前, 应用在 DSSC 中光敏剂的有机染料多为钉基配合物染料 以及卟啉、酞菁类染料等功能染料, 存在价格昂贵、波 长吸收范围小、易团聚等不足 ${ }^{[39]}$. 由于太阳光的总光子 通量约 $60 \%$ 处于大于 $600 \mathrm{~nm}$ 的波长范围内, 约 $50 \%$ 集 中在 $600 \mathrm{~nm}$ 到 $1000 \mathrm{~nm}$ 的可见光和近红外区波长范围 内 $^{[40]}$ ，而克酮酸菁染料从可见光区到近红外都有一定 的吸收，且近红外区吸收强度高、摩尔吸光系数大、光 热稳定性好; 同时具有成本低、分子结构易修饰等优点. 因此，克酮酸菁染料可望作为染料敏化太阳能电池中的 光敏剂，应用前景广阔。

\section{5 其它应用}

除上述应用外, 克酮酸菁染料在二极管激光器的输 出范围内 $(800 \mathrm{~nm}$ 左右 $)$ 有强烈的吸收，而且具有良好的 光热稳定性, 因此在光学记录介质等领域有巨大的应用 优势. 化合物 11 是一种新型的水溶性克酮酸菁染料, 它 可以采用水性旋涂法制作近红外激光系统里的滤光片, 克服了传统旋涂法采用有机溶剂带来的环境危害 ${ }^{[35]}$. 此外, 该类染料独特的光谱特性使其在激光防护领域也 有一定的应用前景.

\section{4 结论与展望}

克酮酸菁染料的最大吸收波长在近红外区，峰型 窄, 摩尔吸光系数高, 苂光发射能力强, 光热稳定性好, 具有光学性能优异、分子结构易修饰等特点, 在光学材 料、生物标记、光动力治疗、太阳能电池等方面应用前 景广阔. 该类染料分子结构中有大的离域 $\pi$ 键体系，其 中包含吸电子基团和给电子基团，可望通过 PariseParr-Pople 分子轨道理论和 TDDFT(含时密度泛函理论) 方法, 研究并设计新型克酮酸菁染料分子结构, 不断调 
节最大吸收波长等分子特性, 使该类染料具备更为独特 的功能, 以满足不同领域的实际应用要求 ${ }^{[41,42]}$.

目前, 国内外关于克酮酸菁染料的报道较少, 主要 还是停留在方酸菁染料的研究阶段. 针对方酸菁染料的 研究, 其技术和研究领域已趋于完善和成熟. 与方酸菁 染料相比, 克酮酸菁染料在光学及光电转换等方面具有 独特的性能和特征, 如非线性光学性能等. 随着人们对 于新型材料的需求, 开发利用克酮酸菁染料已引起人们 的关注. 对于新型克酮酸菁染料的开发和应用还有许多 的领域有待深入研究, 随着新反应、新合成技术的不断 发展和深入, 以及新应用领域的逐步拓展, 克酮酸菁染 料将拥有更为广阔的应用前景, 可望成为功能材料热点 领域研究中的新兴成员.

\section{References}

[1] Fabian, J. Chem. Rev. 1992, 92, 1197.

[2] Fabian, J.; Zahradnik, R. Angew. Chem., Int. Ed. 1989, 28, 677.

[3] Law, K. Y. Chem. Rev. 1993, 93, 449.

[4] Shi, Q. Q.; Sun, R.; Ge, J. F. Dyes Pigm. 2012, 93, 1506.

[5] Deligeorgiev, T.; Kaloyanova, S.; Vasilev, A. Dyes Pigm. 2011, 90, 170.

[6] Elsherbiny, A. S.; Salem, M. A.; Ismail, A. A. Chem. Eng. J. 2012, $200,283$.

[7] Li, Z.-Y.; Xu, S.; Huang, X.; Zhang, F.-S.; Kazuo Kasatani. Photogr. Sci. Photochem. 2007, 25, 241 (in Chinese). (李忠玉, 徐松, 黄新, 张复实, 笠谷和男, 感光科学与光化学, 2007, 25, 241.)

[8] Yang, X.-B.; Ding, S.-T.; Yang, Y.-S.; Zhou, X.-H. Chin. J. Org. Chem. 2002, 32, 34 (in Chinese). (杨小兵, 丁松涛, 杨裕生, 周晓海, 有机化学, 2002, 32, 34.)

[9] Bigelow, R. W.; Freund, H. J. Chem. Phys. 1986, 107, 159.

[10] Srinivas, K.; Prabhakar, C.; Devi, C. L. J. Phys. Chem. A 2007, $111,3378$.

[11] Takechi, K.; Kamat, P. V.; Avirah, R. R. Chem. Mater. 2008, 20, 265.

[12] Yasui, S.; Matsuoka, M.; Kitao, T. Dyes Pigm. 1988, 10, 13.

[13] Kim, S. H.; Hwang, S. H.; Song, H. C.; Yoon, N. S. J. Korean Chem. Soc. 1996, 40, 741.

[14] Tian, M. Q.; Tatsuura, S.; Furuki, M.; Sato, Y.; Iwasa, I.; Pu, L. S. J. Am. Chem. Soc. 2003, 125, 348.

[15] Prabhakar, C.; Chaitanya, G. K.; Sitha, S.; Bhanuprakash, K.; Rao, V. J. J. Phys. Chem. A 2005, 109, 2614.

[16] Prabhakar, C.; Yesudas, K.; Chaitanya, G. K.; Sitha, S.; Bhanuprakash, K.; Rao, V. J. J. Phys. Chem. A 2005, 109, 8604.

[17] Yesudas, K.; Bhanuprakash, K. J. Phys. Chem. A 2007, 111, 1943.
[18] Langhals, H. Angew. Chem., Int. Ed. 2003, 42, 4286.

[19] Meier, H.; Dullweber, U. J. Org. Chem. 1997, 62, 4821.

[20] Meier, H.; Dullweber, U. Tetrahedron Lett. 1996, 37, 1191.

[21] Tatsuura, S.; Tian, M.; Furuki, M.; Sato, Y.; Iwasa, I.; Mitsu, H. Appl. Phys. Lett. 2004, 84, 1450.

[22] Yefimova, S.; Lebed, A.; Sorokin, A.; Guralchuk, G.; Borovoy, I.; Malyukin, Y. J. Mol. Liq. 2012, 165, 113.

[23] Meng, T.; Kang, L.-H.; Zhou, H.; Zhang, X.; Wang, D. J.; Li, X.-W.; Zou, J. Spectrosc. Spec. Anal. 2010, 30, 1362 (in Chinese). (孟涛, 康灵华, 周惠, 张朝, 王笃金, 李晓苇, 邹竞, 光谱学与 光谱分析, 2010, 30, 1362.)

[24] Meng, T.; Kang, L.-H.; Xu, D.-F.; Wang, D.-J.; Li, X.-W.; Zou, J. Spectrosc. Spec. Anal. 2010, 30, 1053 (in Chinese).

(孟涛, 康灵华, 徐端夫, 王笃金, 李晓苇, 邹竞, 光谱学与光谱 分析, 2010, 30, 1053.)

[25] Yang, S.-J.; Tian, H. Photogr. Sci. Photochem. 1999, 17, 275 (in Chinese).

(杨松杰, 田禾, 感光科学与光化学, 1999, 17, 275.)

[26] Sprenger, H. E.; Ziegenbein, W. Angew. Chem., Int. Ed. Eng. 1966, $78,937$.

[27] Zhang, X. H.; Li, C.; Cheng, X. X.; Wang, X. S.; Zhang, B. W. Sens. Actuators, B 2008, 129, 152.

[28] Simard, T. P.; Yu, J. H.; Zebrowski-Young, J. M.; Haley, N. F.; Detty, M. R. J. Org. Chem. 2000, 65, 2236.

[29] Keil, D.; Hartmann, H.; Reichardt, C. M. Liebigs Ann. Chem. 1993, 935.

[30] Kurdiukova, I. V.; Kulinich, A. V.; Ishchenko, A. A. New J. Chem. 2012, 36, 1564.

[31] Avirah, R. R; Jyothish, K.; Ramaiah, D. J. Org. Chem. 2008, 73, 274.

[32] Encinas, C.; Otazo, E.; Laia, R. Tetrahedron Lett. 2002, 43, 8391.

[33] Peng, X.-J.; Wang, L.-Q. CN 1159385, 2004 [Chem. Abstr. 2004, 140, 129771].

[34] Song, X. Z.; Foley, J. W. Dyes Pigm. 2008, 78, 60.

[35] Ramaiah, D.; Eckert, I.; Arun, K. T.; Weidenfeller, L.; Epe, B. Photochem. Photobiol. 2002, 76, 672.

[36] Prabhakar, C.; Yesudas, K.; Bhanuprakash, K.; Rao, V. J.; Kumar, R. S. S.; Rao, D. N. J. Phys. Chem. C 2008, 112, 13272.

[37] Li, Z. Y.; J, Z. H.; Kasatani, K.; Okamoto, H. Phys. B 2006, 382, 229.

[38] Wang, Z.-Y.; Wang, R.-Y.; Fu, N.-Y. Chin. J. Org. Chem. 2011, 31,415 (in Chinese).

(王振宇, 王如勇, 傅南雁, 有机化学, 2011, 31, 415.)

[39] Zhou, D.; She, X.-L.; Song, G.-J. Precious Metals 2010, 31, 37 (in Chinese).

(周迪，余希林，宋国君，贵金属, 2010, 31, 37.)

[40] Rand, B. P.; Xue, J. G.; Yang, F.; Forrest, S. T. Appl. Phys. Lett. 2005, 87, 233508 .

[41] Fabian, J.; Perchert, R. J. Phys. Org. Chem. 2010, 23, 1137.

[42] Fabian, J. Dyes Pigm. 2010, 84, 36 